\title{
Observation of Tissues in Solution by Atmospheric Scanning Electron Microscope (ASEM)
}

\author{
Nassirhadjy Memtily, ${ }^{1,2,3}$ Kazuhiro Mio ${ }^{1,2}$, Mari Sato ${ }^{2}$, Tatsuhiko Ebihara ${ }^{2}$, Chikara Sato ${ }^{1,2}$ \\ 1. Graduate School of Comprehensive Human Sciences, University of Tsukuba. 1-1-1 Tennodai, \\ Tsukuba, Ibaraki Prefecture 305-0006, Japan. \\ 2. Biomedical Research Institute, National Institute of Advanced Industrial Science and Technology \\ (AIST), Umezono 1-1-4, Tsukuba, Ibaraki 305-8568, Japan. \\ 3. Traditional Uighur Medicine Institute of Xinjiang Medical University, 393 Xinyi Rd, Urumqi, \\ Xinjiang Uighur Autonomous Region, 830011 China.
}

Standard electron microscopy (EM) has sub-nm or $\mathrm{nm}$ resolution, but samples must be observed in vacuum. This requires time-consuming pretreatment. However, direct observation of subcellular structures of tissues immersed in solution should be quick and allow high-throughput immuno-EM in order to understand their structure-function relationships. To reduce the time required, we have recently developed an inverted scanning electron microscope (SEM) capable of $8 \mathrm{~nm}$ resolution $[1,2]$. The sample is at atmospheric pressure in a detachable, open culture dish and can be immersed in liquid. A silicon nitride film window in the base of the dish maintains a vacuum in the SEM column and allows electrons to pass through. Backscattered electrons from the sample are captured by a detector under the dish. Using this atmospheric SEM (ASEM), metal-stained and/or immunolabeled tissues up to 2-3 $\mu \mathrm{m}$ from the SiN film were observed.

Mouse tissues (cerebrum, cerebellum, spinal cord, brachial plexus, lung, kidney, liver, esophagus, stomach, intestine, placenta, cardiac and skeletal muscle) were dissected into 1 - $2 \mathrm{~mm}$ thick slabs, fixed with 4\% paraformaldehyde and/or $1 \%$ glutaraldehyde (GA), perforated with $0.5 \%$ Triton X-100, and stained with $2 \%$ Uranyl Acetate or 1-2\% phosphotungstic acid (PTA) and imaged immersed in 10 $\mathrm{mg} / \mathrm{ml}$ glucose solution by ASEM at $30 \mathrm{kV}$ acceleration voltage. Spinal cord and gastrocnemius muscle stained with PTA are shown in Figure 1. In the spinal cord, the nuclei were brightly distinguished above the background. Neurite-like fibers were also stained, although weaker (Figure 1A and B). In gastrocnemius muscle tissue, muscle fibers, motor-end plates, I, A and Z bands were evident together with motor axon-like fibers and neurites (C and D). Commensalism of the epidermal side of stomach with bacteria was also visualized by ASEM (E and F). Immunolabeled tissues in liquid were successfully observed using environmental capsule by Nyska et al [3]. In the present study, perforated spinal cords were tagged with antibody against $200 \mathrm{kDa}$ neuro-filament-H (NF-H), further with FluoroNanogold [4, 5], and observed using ASEM. In the fluorescence image, NF-H is localized mainly in an axon-like filament and its proximal perikarya of neurons (Figure 2A and B). After gold enhancement, white signals from the gold tags were visualized in similar structures by ASEM (C and D). The location in the whole tissue was defined by counter staining with PTA (E and F).

The ASEM is widely applicable not only to biology, but also to drug discovery, pathogen diagnosis, food science, cosmetics, polymer chemistry, nano and material science [6]. The use of an open specimen dish rather than an environmental capsule [7, 8, 3] favors high throughput. Indeed, ASEM has the potential to accelerate intra-operative cancer diagnosis. ASEM can be applied to the in situ study the host-microbe interaction that is critical for homeostasis. In such experiments, tissue culture on the open ASEM dish can be externally manipulated or doped with drugs while being observed by microscopy. 


\section{References}

[1] H Nishiyama et al, J Struct Biol. 172 (2010), p. 191-202.

[2] C Sato, JEOL news 46 (2011), p. 17-22.

[3] A Nyska et al, Experimental \& Toxicologic Pathology. 57 (2006), p. 291-297.

[4] Y Maruyama et al, J Struct Biol. 180 (2012), p. 259-270.

[5] T Murai et al, Int J Mol Sci. 14 (2013), p. 20809-20819.

[6] M Suga et al, Ultramicroscopy 111 (2011), p. 1650-1658.

[7] S Thiberge et al, PNAS. 101 (2004), p. 3346-3351.

[8] V Behar, Microscopy \& Analysis. 108 (2005), p. 21-23.

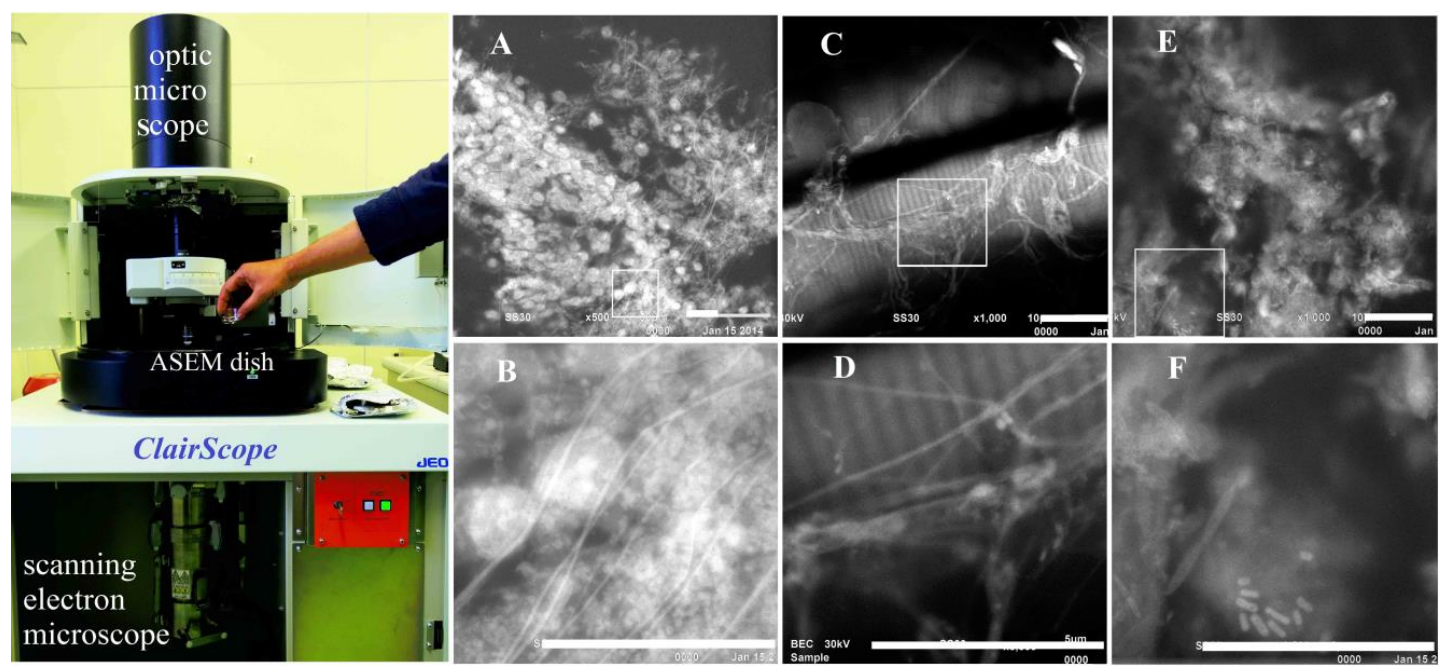

Figure 1. ASEM of mouse spinal cord and gastrocnemius muscle. (A) Spinal cord stained with PTA. (B) Higher magnification of the white rectangle in the left panel. Nuclei and neurites up to 2-3 $\mu \mathrm{m}$ from the SiN film were visualized. (C and D) Gastrocnemius muscle stained with PTA. (E and F) Stomach image indicating commensalism with bacteria. Scale bars: $20 \mu \mathrm{m}$.

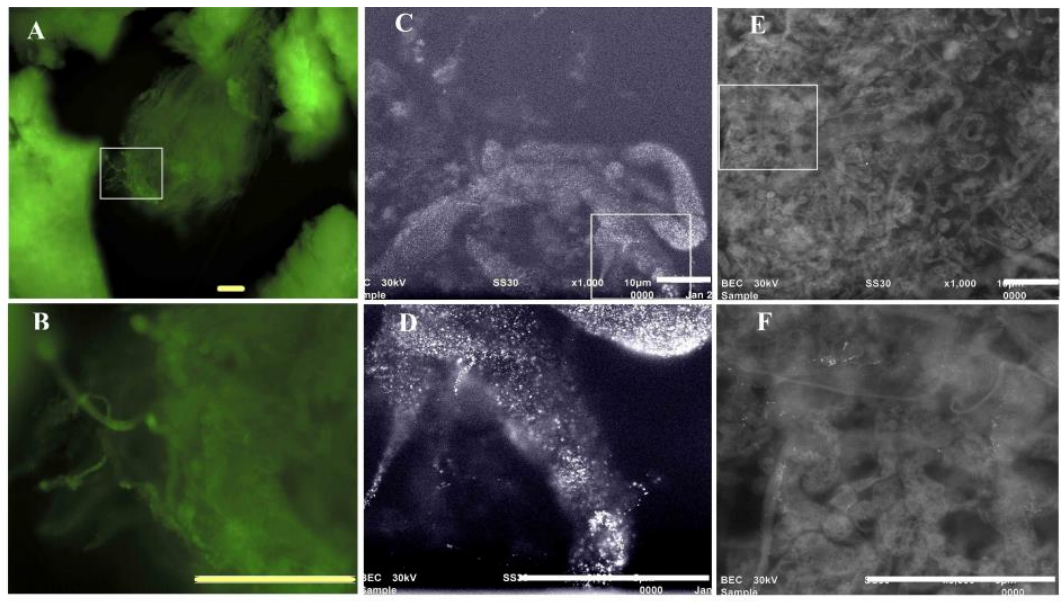

Figure 2. ImmunoEM of neurofilament-H (NF-H) in spinal cord. NF-H was tagged with FluoroNanogold. (A) Fluorescence microscopy. (B) Higher magnification images of the white rectangle in the upper panel. (C and D) ASEM after gold enhancement. ( $E$ and F) Counter stained with PTA. NF-H is mainly localized in an axon-like filament and its upstream perikarya of neurons. Scale bars: 20 $\mu \mathrm{m}$. 